\title{
POST-PROCESSING OF TRANSFORM CODED IMAGES USING SELECTIVE FILTERING AND FUZZY-BASED ENHANCEMENT
}

\author{
K.T. Choi, S. C. Chan, and T. S. Ng \\ Department of Electrical and Electronic Engineering \\ The University of Hong Kong \\ email: scchan@hkueec.hku.hk
}

\begin{abstract}
Transform coding often leads to artifacts called blocking when the image or video is compressed to low bit-rate. In this paper, a new post-processing scheme using selective low-pass filtering and fuzzybased enhancement is proposed. The merit of the scheme is that it can remove most of the blocking artifacts found in image or video coding while preserving most of the fine details. Simulation results demonstrated that the proposed algorithm can significantly improve the visual quality of the decoded image.
\end{abstract}

\section{INTRODUCTION}

Transform coding using the Discrete Cosine Transform (DCT) is the most popular approach for image compression. However, it often leads to artifacts called blocking when the image or video is compressed to low bit-rate. This artifact can be reduced by proper selecting the quantization step sizes in the coder and/or by post-processing the decoded image. Blocking artifact originates from the block based nature of transform coding. Since the image is divided into square blocks and each block is coded and quantized individually, there will be noticeable discontinuity between adjacent blocks when the picture is encoded at low bit-rate. Since blocking is a high frequency artifacts, low-pass filtering can be used to reduce its effect. However, simple low-pass filtering usually leads to over-smoothing of the high frequency components found in edges and textures.

Previous research on post-processing using spacevariant filters [1] was done by smoothing of the edges and by estimating the edge information in the compressed image data [2]. This is a rather difficult in low bit-rate coding since the actual edge information will be severely distorted. Other approach based on iterative method has also been proposed. In [3], the image is reconstructed using the method of projections onto convex sets (POCS) [3] where a number of constrains on the coded images such quantization stepsize are used together with low-pass filtering to remove the blocking artifacts.
In this paper, a new post-processing algorithm using a two stages approach is proposed. The method is based on simple low-pass filtering on smooth area, followed by edge enhancement using nonlinear filtering . In the first stage, we selectively perform lowpass filtering on the smooth blocks in the image. This involves the classification of edge/texture and smooth blocks in the image. In the second stage, we apply fuzzy rule-based nonlinear edge enhancement to recover the details that will be lost in the final filtering process. The merit of the scheme is that it can remove most of the block artifacts found in the image or video while preserving most of the fine details. Simulation results demonstrated that the proposed algorithm can significantly improve the visual quality of the decoded image. The layout of the paper is as follows: In section II, we shall briefly discuss the basic idea of our approach. The classification algorithm and the fuzzy rule-based enhancement algorithm will be discussed in Section III and IV, respectively. The performance of the proposed algorithm will be given in Section V.

\section{PROPOSED POST-PROCESSING METHOD}

Our post-processing algorithm consists of two different stages:

1. Detection of texture/edge and smooth areas and perform low-pass filtering on the smooth region.

2. Fuzzy-based image enhancement to improve the subjective quality.

The purpose of the first stage is to remove the blocking artifacts of the image in the smooth, flat region in which blocking effect is most perceivable. Due to the low-pass filtering, the resulting image will be blurred and some high frequency components such as edges are removed. In order to recover the sharpness of the overall image, we enhance those smoothed edges by a fuzzy-based enhancement process.

\section{DETECTION OF THE SMOOTH AREA}

To remove the blocking artifacts in the encoded image, we can apply low-pass filtering to smooth out these block discontinuities. However, the resulting image 
will also be blurred due to the smoothing of the edges and other texture regions. In the proposed algorithm, we first classify whether a given pixel is belonged to a smooth or a texture/edge area. Those regions which are classified as smooth area are then filtered by the low-pass filter. Using this classification approach, we can filter those blocks within the smooth region, while keeping the texture/edge regions untouched.

The classification procedure first divides the whole picture into $(8 \times 8)$ blocks and calculate the mean, $m$, of each block:

$$
m=\frac{1}{64} \sum_{x=0}^{8} \sum_{y=0}^{8} f(x, y)
$$

The absolute difference between each pixel value and the mean is calculated. If it is less than a given threshold i.e.

$$
|f(x, y)-m|<T_{1}
$$

where $f(x, y)$ is the pixel value at position $(x, y)$, it is classified as a smooth pixel. The threshold $T_{1}$ is a variable determined by taking into account the contrast sensitivity of the human visual system according to the Weber's law:

$$
T_{1}=\eta m
$$

where $\eta$ is a stringency factor introduced to regulate the proportion of pixels being classified as smooth pixels and is chosen to be 0.1 . If the number of smooth pixels in a given block exceeds certain threshold, $T_{2}$, then the block is classified as a smooth block. Otherwise, it is classified as an edge/texture block which is left untouched.

After the classification, we can filter those blocks that are classified as smooth area so as to remove the blocking artifacts. The filter that we used is a $(5 \times 5)$ finite impulse response (FIR) filter used in [3]. Its impulse response is shown in Fig. 1.

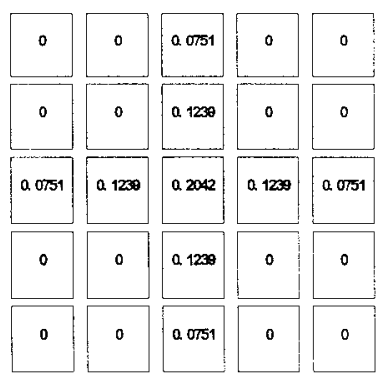

(a)

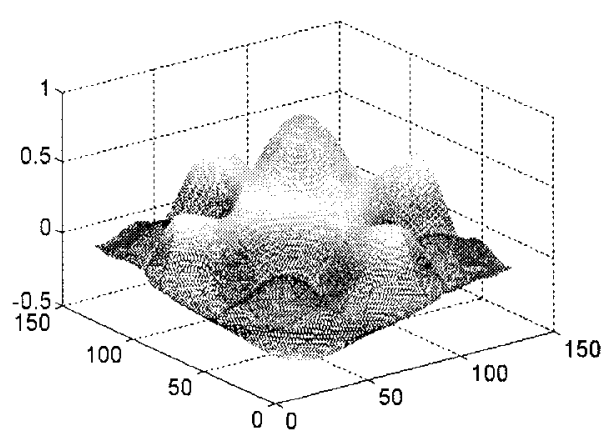

(b)

Figure 1. FIR filter used for low-pass filtering (a) the impulse response and (b) Frequency response.

This filtering process can remove most of the block artifacts, without introducing excessive blurring in the image. However, it was found that filtering the image by the above process once cannot remove the artifacts completely. Therefore, we have to apply the filtering process repeatedly to the smooth region of the image. In our simulation, the Lena image is filtered 3 times and the resulting image is almost free from blocking artifacts. The image is slightly blurred and the fuzzybased enhancement will be applied to improve the visual quality of the encoded image.

\section{FUZZY-BASED ENCHANCEMENT FILTER}

Fuzzy-based enhancement of images have been proposed in $[4,5]$. These rule-based fuzzy operator is a new class of nonlinear operators that can be applied to digital image processing. The advantages of Fuzzybased enhancement are their low implementation complexity and noise-insensitive sharpening. Here we shall apply this technique to recover part of the details that have been lost in the low-pass filtering.

\section{A. Fuzzy System Operation}

In this section, we shall briefly describe the principle of the fuzzy system. Fig. 2 illustrates the flow of data through a fuzzy system. The system consists of three different parts: fuzzification, rule evaluation and defuzzification. In the fuzzification stage, a predefined membership functions is used to map each system input variables into one or more degree of membership. Fuzzification is the process of calculating a value to represent an input degree of membership in the fuzzy set. Then the predefined rules in the rule base are evaluated by combining degrees of membership to form output strengths. The inference method (see Fig. 3) used is called Min-Max 
Method. The rule operation has an IF-THEN structure. Each rule includes a group of conditions (antecedent clauses) about input variables and specifies an action to the output variable. The fuzzy rule relating the input and output variables are expressed in following form:

IF (Antecedent I is True) AND (Antecedent 2 is False) AND

(Antecedent $N$ is True)

THEN (Consequent is done)

Lastly, the defuzzification stage computes system outputs based on the strengths and membership functions. In this stage, the output of the fuzzy system should be a scalar value, and a typical defuzzication method called gravity center method is used here due to their low implementation complexity.

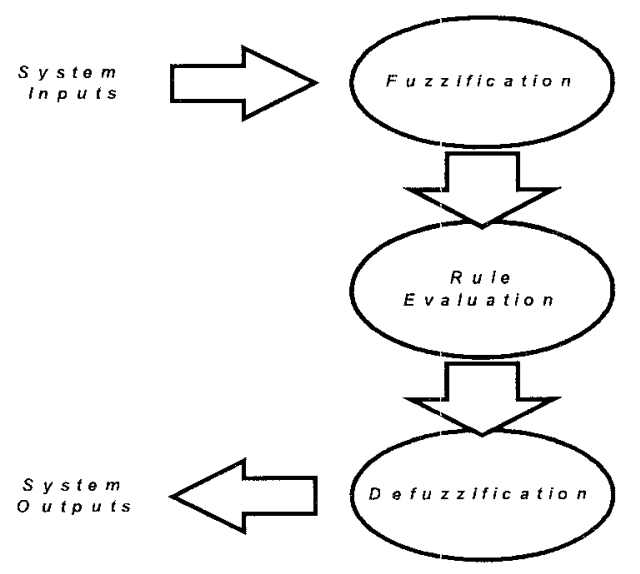

Figure 2. Operation of a fuzzy system.

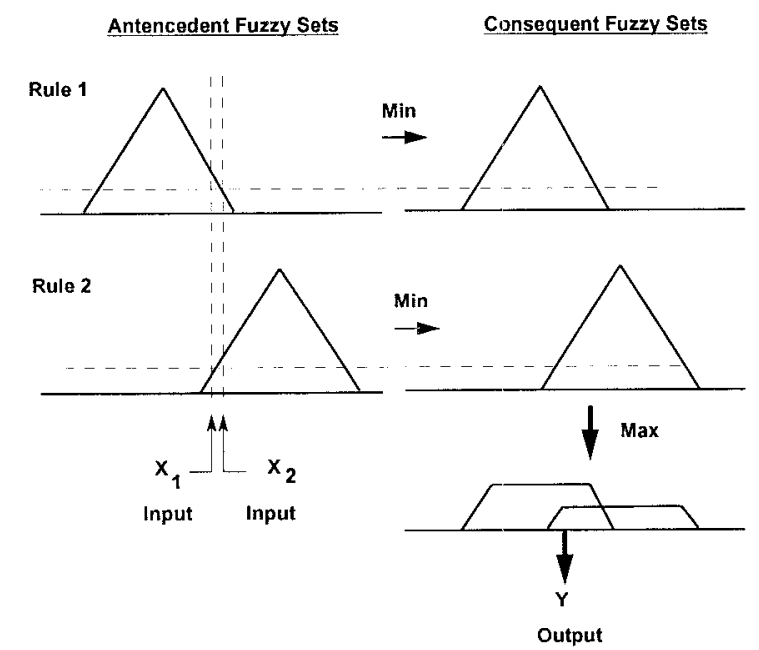

Figure 3. The fuzzy inference method for image enhancement.

B. Enhancement Fuzzy Process
Let $P_{0}$ be a pixel in the input image and the neighborhood pixels are:

\begin{tabular}{|l|l|l|}
\hline$P_{1}$ & $P_{2}$ & $P_{3}$ \\
\hline$P_{4}$ & $P_{0}$ & $P_{5}$ \\
\hline$P_{6}$ & $P_{7}$ & $P_{8}$ \\
\hline
\end{tabular}

The input variables are the luminance differences between the current pixel $P_{0}$ and the neighborhood pixels:

$$
X_{j}=P_{j}-P_{0}
$$

where $\mathrm{j}=1, \ldots, 8$. The output variable $\mathrm{Y}$ is a luminance difference and represents a correction term which is then added to $P_{0}$ in order to enhance the image.

$$
P_{0}^{\prime}=P_{0}+Y
$$

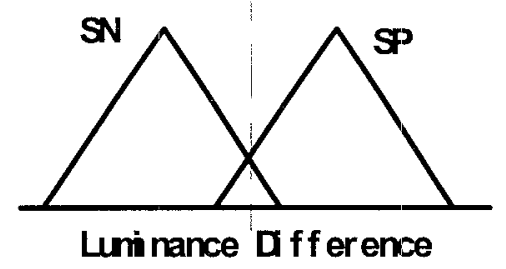

(a)

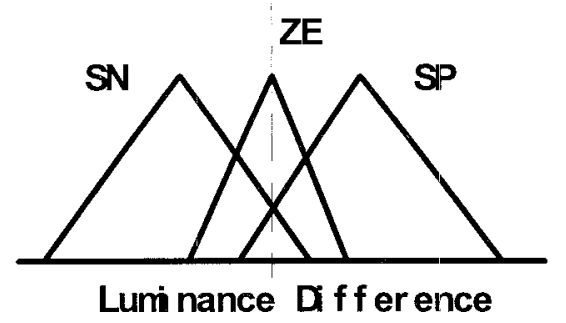

(b)

Figure 4 (a) Antecedent and (b) Consequent fuzzy sets of the fuzzy based filter proposed.

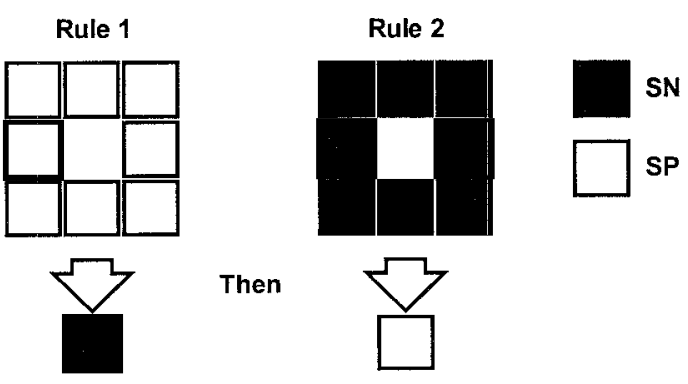

Figure 5. Graphical notation of Fuzzy Rules for image enhancement. 
The membership function we used is the triangular shaped fuzzy sets as shown in Figure 4. In our case, the fuzzy rule(s) for edge enhancement should be:

$$
\begin{aligned}
& \text { IF present pixel is much brighter (or darker) } \\
& \text { than the neighboring pixels, } \\
& \text { THEN increase (reduce) the luminance of the } \\
& \text { pixel. }
\end{aligned}
$$

In our fuzzy based filter, each fuzzy set is defined by a piecewise linear membership function. The fuzzy sets for the antecedents and consequent used are shown in Fig. 4. The sharpening rules are shown in Fig. 5. The idea behind the rules in Fig. 5 is to increase the local contrast of the data by amplifying the differences between the present pixel and its neighbors. On the uniform smooth area, the luminance difference should be small, and the sign of the input variable is random. Due to the small overlap between the Small Positive (SP) and Small Negative (SN) fuzzy sets, both rules are activated to the same extent, and hence the output value of the correction is also small. On the image details, there is a sharp change in the luminance differences within the window, making some input variables larger than the others. Thus, one of the rules would dominate, and the correction value would further increase this luminance difference.

\section{EXPERIMENTAL RESULTS}

The proposed post-processing algorithm had been applied to a JPEG compressed lena image at $0.2 \mathrm{bpp}$. There are lot of blocking artifacts in the image (Fig.6a). Fig. 6b shows the post-processed image. It can be seen that most of the blocking artifacts have been removed and the details in the texture and edge areas are well preserved. Some fine texture in the hat had been removed due to the difficulty in classification of texture/edge area in the region. Also, a final filtering had been applied to the whole picture resulting in some lost of fine details in the picture. Nevertheless, the overall visual quality of the image is significantly improved with almost the same brightness and sharpness as the decoded image.

\section{CONCLUSION}

A post-processing scheme for transform coded images using selective filtering and fuzzy-based enhancement is presented. It removes most of the block artifacts found in low bit-rate image or video while preserving most of the fine details in the decompressed picture. Simulation results demonstrated that the proposed algorithm can significantly improve the visual quality of the decoded image.

\section{REFERENCES}

[1] B.Ramamurthi and A.Gersho, "Nonlinear Space-Variant Postprocessing of Block Coded Images." IEEE Trans. ASSP, vol.34, no. 5 , pp. 1258-1268, Oct. 1986

[2] K.Sauer, "Enhancement of Low Bit-Rate Coded Images Using Edge Detection and Estimation," Computer Vision Graphics and Image Processing: Graphical Models and Image Processing, vol.53, no. 1, pp.52-62, Jan, 1991.

[3] A.Zakhor, "Iterative Procedures for Reduction of Blocking Effects in Transform Image Coding," IEEE Trans. Circuits Syst. Video Technol., Vol. 2, pp.91-95, Mar.1992.

[4] F. Russo and G. Ramponi, "Fuzzy operator for sharpening of noisy images," IEE Electron. Letter, vol, 28 , 1992, pp. 1751-1717.

[5] F. Russo and G. Ramponi, "A Fuzzy Operator for the Enhancement of Blurred and Noisy Images," IEEE Trans. IP, vol. 4, No., 8, pp.1189-1174, Aug. 1995.

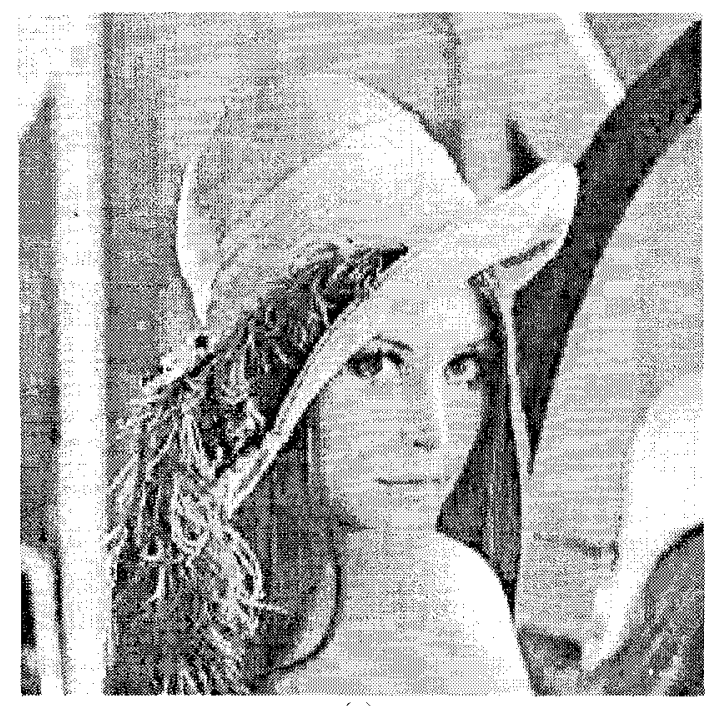

(a)

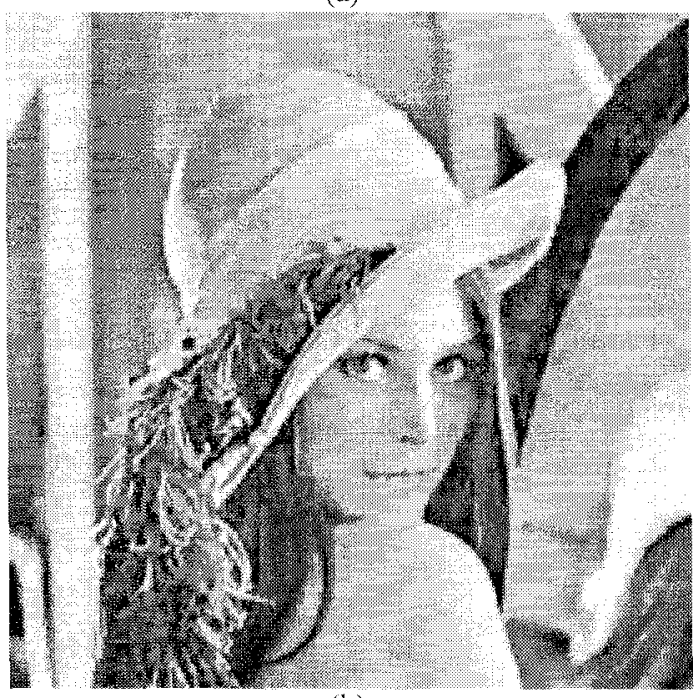

(b)

Figure 6. Image Lena with size $(512 \times 512)$ (a) compressed with JPEG at $0.2 \mathrm{bpp}$ (b) the Post-processed image. 